\title{
Recruitment Strategies Used in a Survey of African Immigrant Maternal Mental Health in Alberta, Canada
}

\author{
Chinenye Nmanma Nwoke ${ }^{1}$ (D) $\cdot$ Oluwagbohunmi Awosoga ${ }^{1} \cdot$ Brenda MY Leung $^{1}$
}

Received: 29 March 2021 / Revised: 24 May 2021 / Accepted: 26 May 2021 / Published online: 7 June 2021

(C) W. Montague Cobb-NMA Health Institute 2021

\begin{abstract}
African immigrant women are underrepresented in health research on maternal mental health. Thus, there is a need to highlight successful recruitment strategies to engage African women in health-oriented research. This paper offers insights on recruitment strategies utilized in recruiting African immigrant women in Alberta (Canada) with infants 2 years of age or under for a survey study on maternal mental health. We recruited 136 African immigrant women. Most participants were recruited by using already established social networks in the community. Other successful strategies included referral from community partners (i.e., immigrant organizations, cultural association, religious institutions), participants, utilizing an online survey tool (i.e., Qualtrics), and through family and friend networks (i.e., word-of-mouth). This study evidently highlights the importance of utilizing multiple recruitment strategies to successfully meet the desired sample size for a survey study. We believe the lessons learned during the process of recruitment will be helpful for others working with other African immigrant women populations in Canada and in other Western societies.
\end{abstract}

Keywords African immigrants $\cdot$ Maternal mental health $\cdot$ Recruitment strategies $\cdot$ Perinatal mental health

\section{Introduction and Purpose}

African immigrants are on the rise in Canada. In census statistics between 1996 and 2001, the number of African immigrants grew by $32 \%$, whereas the overall Canadian population only grew by $4 \%$ [1-3]. By 2016, $13.4 \%$ of recent immigrants to Canada were born in Africa, underlining a four-fold increase from the 1971 census where only $3.2 \%$ of recent immigrants were from Africa $[4,5]$. Africa thus ranked second, ahead of Europe, as a source continent of recent immigrants to Canada. Noteworthy, where there is a lack of equal and adequate representation in any population-based research, there is also a lack of diverse thought which perpetuates health disparities by limiting strong evidence base to a non-diverse population.

African immigrants tend to be underrepresented in national health surveys (i.e., the Canadian Community Health Survey) leading to a gap in understanding the health needs of African

Chinenye Nmanma Nwoke

chinenye.nwoke@uleth.ca

1 Faculty of Health Sciences, University of Lethbridge, 4401 University Drive, Lethbridge, AB T1K 3M4, Canada immigrant women in Canada [6-8]. This is in part due to the challenges associated with engaging African immigrants in research; investigators conducting research with African immigrants, and other immigrant groups, often encounter substantial challenges in recruiting and gaining the trust of participants $[9,10]$. Even with the development and implementation of some innovative research approaches (i.e., community partnerships, involving community members in the research process, direct contact between participants and the research team at events) by health researchers to improve recruitment and retention of immigrant populations in Canada [10,11], we do not know if these strategies are effective in the recruitment of African immigrant women with infants.

Successful recruitment and retention methods will result in the inclusion of a higher percentage of African immigrant women in Alberta and Canada into future epidemiologic research on maternal mental health in the perinatal period. This could result in a more accurate understanding of the patterns and trajectories of the mental health of African immigrants before and after their arrival to Canada. In addition, the inclusion of African immigrants into maternal mental health studies is fundamental to our understanding of the influence that acculturation, acculturation-related stress, and other social determinants have on the mental health outcomes of African immigrants as a whole. 
In this paper, we report the methods of recruitment of African immigrant women for a cross-sectional study on immigrant maternal mental health in Alberta, Canada, in 2020. In addition, we discuss strategies for recruitment, as well as lessons learned to inform future research studies in this population and facilitate the recruitment of African immigrant women for health research in Canada.

\section{Recruitment Process}

An inventory of African cultural and religious organizations and an inventory of newcomer/immigrant-service organizations in Alberta were compiled through an environmental scan of key agencies, organizations, and institutions with African immigrant memberships. Next, a study introduction letter and recruitment memo were sent via email to the presidents or directors of the respective organizations/associations, to solicit study participants through membership lists. Organizations and/or associations were also asked to post a request for participation in the study on their websites and newsletters, using the study poster, as well as forward the recruitment memo via email to their membership list. Of note, study participants were not contacted directly, but rather through community, religious organizations, and cultural associations across Alberta. Interested postpartum African women then contacted the researcher directly by phone or email.

Additionally, study posters were posted across the key traffic areas at the University of Lethbridge campus, in African groceries store in Calgary and Edmonton, African hair stores and salons across Alberta, and in some African community WhatsApp women groups as added recruitment strategies. Already existing and established social networks within the African immigrant community in Alberta were utilized. These included religious WhatsApp women groups, new immigrant WhatsApp groups, transitioning African immigrant professionals WhatsApp groups, African professional development Facebook groups, African cultural association Facebook groups, LinkedIn, Instagram, and Twitter. In all instances, the study recruitment flyer was posted weekly throughout the recruitment time period. Study recruitment wording was revised to more non-academic and succinct language. Furthermore, study information booths were to be set-up at summer community or cultural events in major cities in Alberta - Calgary, Edmonton, and Lethbridge - as it is assumed that most major immigrant community events occur more frequently in bigger cities like Calgary and Edmonton and attract a greater number of immigrants, including African immigrants.

Once a potential participant expressed interest in the study, eligibility was assessed. A four-question pre-survey screening (inclusion criteria) was sent to the participant via the respective method they used to contact the researcher, i.e., via email for those that expressed interest via email, or text message for those that expressed interest via text message, to determine inclusion or exclusion from the study. The four questions are the following: (1) I am aged 18 years or older; (2) I reside in the province of Alberta; (3) I had a live birth, and my infant is aged 2 years or younger at the moment; (4) I identify as African. Once a respondent answers "yes" to all four questions, they were deemed eligible to participate in the full study.

Following this, eligible participants were automatically redirected to the online study questionnaire through the Qualtrics platform, or request for a paper questionnaire package. In both instances of questionnaire administration, before starting the data collection, informed consent was obtained. Online participants were allowed to give consent via the online consent form, whereas paper-based participants were mailed a consent form along with their questionnaire package. All terminology used in the study questionnaire (i.e., depression, anxiety, mental health) were meticulously defined.

Participants were asked to recommend the study to others they know that will meet eligibility for the study. This information was communicated to all participants in the recruitment memo and also on the study questionnaire. Participants were asked to share the principal investigator's email and telephone contact information with potential study participants, and online participants were also asked to forward the recruitment memo email to their network of contacts who may be interested in participating in the study.

For eligible participants, a 63-item questionnaire was utilized to collect information. This questionnaire begun with a section on informed ethical consent and then was sub-divided into sections corresponding to the determinants of population health framework, namely sociodemographic variables, socioeconomic variables, social network variables, health status variables, and psychosocial/acculturation variables. The dependent variables of the survey study were maternal mental health outcomes, namely maternal depression, measured using the validated EPDS-10 scale, and maternal anxiety, measured using the validated GAD-7 scale. As an incentive for retention, all participants were entered into a raffle draw to win one of five $\$ 20$ gift cards.

A flowchart of the study recruitment process is outlined in Fig. 1:

\section{Ethics Approval}

This study was approved by the Human Participant Research Committee (HPRC) at the University of Lethbridge. Each participant signed a consent form prior to accessing the study questionnaire. Participant responses were anonymous, and they were free to respond or not to any question and were free to withdraw from the study at any time. 
Fig. 1 Flowchart of the study recruitment process

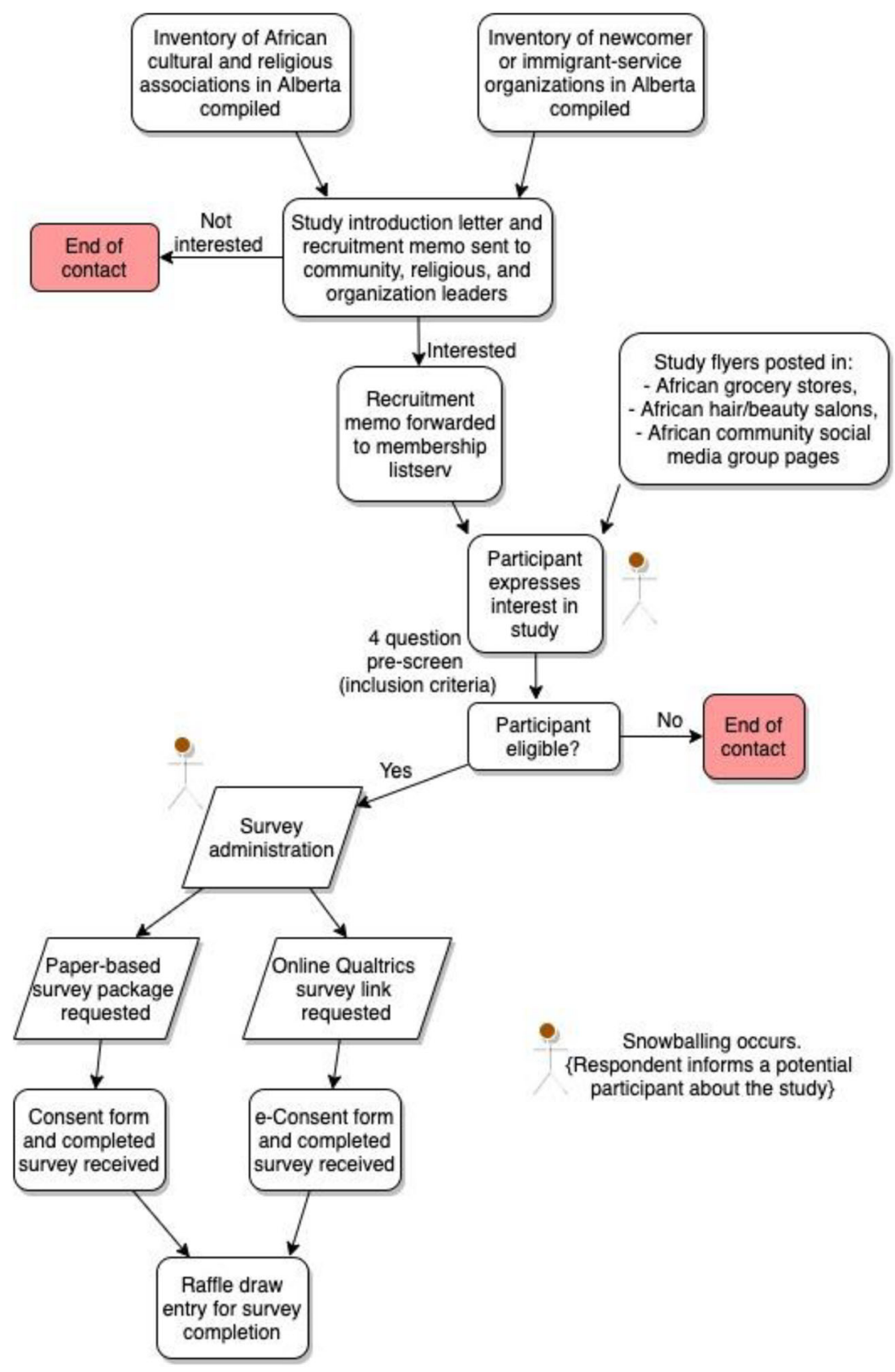

\section{Results}

The study reached 136 African immigrant women in Alberta with infants 2 years of age or under, during the 1-year recruitment period, by using both community-based and social network-based recruitment strategies. These included mobilizing African community and religious institutions, utilizing word-of-mouth through family and friends, ensuring confidentiality through the consenting and anonymized data collection processes, and providing multiple options for data collection —online or paper-based administration.

\section{Successful Recruitment Strategies}

Recruitment in maternal health research can be challenging, particularly for racialized immigrant groups $[12,13]$. In our study, four strategies that were most successful in engaging and recruiting participants were as follows: (1) using social media networks/groups; (2) use of community organizations and associations' networks; (3) using an online survey; and (4) utilizing word-of-mouth through friends and relatives. Of note, the metric used to determine the success of each of the aforementioned recruitment strategies was the number of 
participants engaged and recruited, as evaluated by the expression of interest email and/or text message.

Use of Social Media Networks Utilizing African community social media group pages (i.e., WhatsApp, Facebook, Instagram, Twitter) was one of the most effective recruitment strategies as two-third of participants were recruited through this method. Vital to the success of this strategy was to engage group "gatekeepers" in building trust with potential participants and increase interests about the study. Among African immigrant women in Alberta, gatekeepers included religious leaders (pastor's wives, female church elders), WhatsApp group administrators, and female community leaders. We maintained collaborative relationships with community gatekeepers to increase interest in the study and maximize recruitment. To ensure that "gatekeepers" understood the benefits of the study, we briefly addressed the different groups that had expressed an interest in our study during scheduled meetings to provide a brief overview. For instance, when we sought to recruit participants from churches, we contacted the pastor of the church using information posted online on respective websites and scheduled a meeting to discuss the details of the project. To build trust with gatekeepers, we were fully transparent about our intentions, the details of study, and its benefits to the African immigrant community. We also ensured continuous engagement with gatekeepers through frequent telephone calls, in-person meetings, virtual meetings, and email correspondence to increase buy-in and trust of the research process.

Furthermore, we discussed the importance of the study and how information gleaned through research could improve the health of the African immigrant population, assured gatekeepers of confidentiality and anonymity in data collection, and allowed time for questions. This helped gatekeepers become receptive to the study and helped to ascertain the best days to post study recruitment flyer on their social networks (i.e., online groups). Gatekeepers also helped advertise the study by distributing study flyers at their respective virtual events and invited the researcher and recruitment team to address their groups directly during virtual events. Nonetheless, there were some institutions that declined to participate in our study because of time commitment and lack of interest.

\section{Use of Community Organizations and Associations' Networks} One-third of the study participants were recruited through community-based recruitment with the assistance of a trusted community leader, religious leader, and through snowballing. What was most effective of this strategy was the referral of prospective participants by trusted leaders. The open lines of communication about the importance of a research study of this nature were also proved to be effective in increasing the number of participants recruited through community leaders. Furthermore, the use of community networks, as well as of a strategy to identify the research team during events, gave the team visibility and reinforced a sense of trust in the community. Building relationships of trust with community members is vital to increasing participant recruitment [9-11]. The research team adopted a collaborative approach and established reciprocal relationships with community members (religious leaders, business people, and the leadership of community organizations).

Noteworthy, recruitment through this channels were slow and challenging at times. Some community organizations saw no benefit in collaborating with the study, as they were unaware of the difficulties African immigrant women faced postchildbirth and preferred to protect their members from the study. Ultimately, while most were receptive to assisting, this strategy was only useful in providing a platform for distributing information about the research study.

Use of Online Survey Initially, study recruitment was intended to occur through online methods and in-person recruitment at community events. However, due to the coronavirus disease (COVID-19) pandemic, the online recruitment method proved to be the primary recruitment method. This, in addition to utilizing an online survey tool on the Qualtrics platform, proved to be a successful recruitment strategy. Qualtrics, a web-based survey tool, was chosen because it is an encrypted data management system which enhances participants' confidentiality, is easy to use, and provides a unique, shareable web link to the online survey. Once a participant expressed interest in the study, it was very effective and straightforward to send them an online Qualtrics link to complete the study survey. To ensure survey completion, we asked participants who finished the surveys online to provide their contact information at the end of the survey so that they can be entered into a draw for a raffle to compensate them for their time.

Nonetheless, there were some participants who noted that they had no access to internet services to complete the survey using the online Qualtrics platform. In this instance, approximately seven paper surveys were mailed out. These surveys, although sent with postage-paid return envelopes, proved difficult to follow up with as potential participants may have gotten busy with other priorities, or the community representative in few instances was unwilling to provide contact information for participants as they wanted to remain anonymous (no address/phone/email contact), and thus any opportunities for follow-up were not possible. In future studies, understanding the socioeconomic status (i.e., income - access to internet services) of potential participants is vital. In this respect, future studies could explore the utilization of study recruitment iPads or laptops housed at community centers or cultural association offices, to allow for participants with no internet services to freely access and complete the study survey.

Utilizing Word-of-Mouth Through Friends and Relatives Within many African communities, it is the norm for female 
friends and families to share health information through informal or oral communication $[14,15]$. In this aspect, word-ofmouth (i.e., snowballing) was one of the successful strategies used to recruit study participants. Potential study participants and community members were asked to share information about the study with their close female friends, family members, and other relatives. Later on, the word-of-mouth recruitment strategy advanced to include encouraging study participants to share the direct Qualtrics link for the study with other African immigrant women within their social circles. This allows for potential participants to self-screen and determine eligibility or exclusion from the study. Some participants noted that they were referred through family/friends in the final section of the study survey that allowed for any additional comments in free-text format.

Furthermore, word-of-mouth was a successful recruitment strategy as it could be applied throughout all phases of the study recruitment and allowed for both the source and the recipient to further share recruitment information to a much wider audience/network. Accessibility is high when the information is easily retrieved from memory and shapes explanation [16]. In this respect, the sharing of study information from memory, in lay language via a familiar source, was more likely to be received as credible, truthful, and trustworthy. Direct involvement in the community, with the principal investigator being an African immigrant woman, was also crucial to enable this strategy. Nonetheless, the research team was unable to control the messaging or narrative, and credibility was left to the source of the information. Regardless, we were not informed of any issues or concerns pertaining to this recruitment strategy.

\section{Challenges}

Throughout the study recruitment period, we identified some challenges in fear of stigmatization and some issues related to community partnerships. Some community associations were hesitant in referring their eligible membership to participate in the study as they believed stigmatization could occur. This is because mental health is stigmatized in many cultures [17-19]. To mitigate this challenge, paper copies of the study questionnaire package were placed in discrete locations in community associations and included postage-paid return envelopes. This allowed for interested women to complete the study without any ties to their community associations referring eligible membership. Our experience is not unique and mirrors that of other researchers who have engaged in mental health research with immigrant communities and found stigmatization to also be a key challenge $[11,17]$.

Additionally, our study highlights another challenge where two organizations that declined to participate expressed concerns about how the data collected will be utilized. To mitigate this challenge, we emphasized that the study is ethically approved and that we were bound by the requirements and principles of ethics thought-out the study (i.e., ensuring participant confidentiality and anonymity). We also noted that the principal investigator was also an African immigrant woman with similar experiences to women in the community. As a result of this racial/gender concordance, one of the organizations reconsidered and later agreed to engage its eligible membership in participating in the study. The second organization still declined to share the study information with its membership listserv but invited us to attend an in-person event to talk about the study; this event was later cancelled due to the restrictions as a result of the coronavirus pandemic.

\section{Discussion}

Effective recruitment strategies are essential to the overall success of health research, as low levels of participation by African immigrants are often attributed to a lack of trust in researchers because of historical breeches of ethical research conduct $[20,21]$. This paper reported on four recruitment strategies utilized in a survey study on maternal mental health of African immigrant women in Alberta, Canada, highlighting the success in each strategy, challenges encountered, and mitigation strategies. Online recruitment via already established African social networks (i.e., closed WhatsApp groups) was one of the most promising strategies because it built on existing information sharing norms within the African community in Alberta. The success of this strategy is similar to that seen in other studies on recruiting immigrants for health research $[11,22]$.

Additionally, race/ethnicity concordance was a facilitator of participant recruitment [23, 24]. The principal investigator being an African immigrant woman helped to build trust among the community members and effectively communicated our commitment to improve the maternal mental health of the population. Inclusive language (i.e., "us") was used to collectively refer to the African immigrant women, and we adapted respectful cultural intonations when engaging with community and religious elders. This helped to consider the principal investigator as a "cultural insider." The position of the insider and outsider is a vital concept for cross-cultural and sensitive research [25]. Because of the cultural commonalties, cultural insiders may be better positioned to develop rapport and closer relationships with local people and communities than outsiders $[25,26]$. This could enable African immigrant women to respond to sensitive issues (i.e., maternal mental health) in a timely and appropriate manner. This strategy also facilitated engagement, recruitment, data collection, and ultimately the return of results. Future studies among African immigrant women in Alberta, Canada, as a whole should always include research personnel who share similar African 
heritage to ensure successful recruitment and retention outcomes.

Moreover, the added benefit of initially establishing rapport with the African immigrant community and identifying key gatekeepers, as well as the initial group of participants who serve as the first seeds of snowballing, should not be over-looked. Some studies have shown that the sharing of health information among female friends and families through informal oral methods is a common practice within the African community $[14,15]$. As such, we utilized snowballing through word-of-mouth as an added recruitment strategy building on existing social networks in the community. All study participants were asked to share the study information with their close female relatives and mummy groups. Later on, the snowballing recruitment strategy evolved to include encouraging study participants to also share the web link of the study information with other African immigrant women within their social circles.

Ultimately, this study was able to recruit African immigrant women in a study to understand the prevalence, associated risk factors, help-seeking behaviors, and effects of maternal depression and anxiety disorders within 2 years of giving birth by using a variety of recruitment strategies. Immigrants are a heterogeneous group, and attempts to examine maternal mental health disparities in immigrants in Canada fail to acknowledge the social and cultural heterogeneity, which result in knowledge gaps. Importantly, this paper adds to the body of scholarship on participant recruitment models as it provides evidence based on the success and added value of utilizing multiple strategies in recruiting African immigrant women, an understudied demographic, for maternal mental health research. For future research involving African immigrant women with young infants, using multiple recruitment strategies including already established social networks (i.e., WhatsApp groups, snowballing) to recruit study participants is a promising strategy to build upon.

\section{Conclusion}

In summary, this paper is one of the first attempts to identify explicit strategies and procedures for the recruitment and retention of African immigrant mothers in Alberta with young infants in a quantitative survey study investigating their risk factors and protective factors for maternal depression and anxiety following childbirth. The process provided valuable insights into recommendations for recruiting African immigrant women in quantitative research. Researchers should understand the cultural norms and values, as well as identify preexisting community and social networks in the community as essential factors to the recruiting Africa immigrant women. The recruitment strategies discussed in this paper has contributed to the conduct of our study on maternal depression and anxiety disorders within 2 years of birth among African immigrant women in Alberta Canada.

Acknowledgements The authors wish to thank the African immigrant women across Alberta, religious leaders, gatekeepers, newcomer organizations, cultural associations, and community members for their support and participation in this study.

Availability of Data and Material Not applicable.

\section{Declarations}

Ethics Approval University of Lethbridge HPRC approved the present research on December 11, 2019 (HPRC Protocol Number 2019-116).

Conflict of Interest The authors declare no competing interests.

\section{References}

1. Lindsay, C. (2001). Profiles of ethnic communities in Canada. The African Community in Canada. Analytical Paper. Statistics Canada, Catalogue no. 89-621-XIE-No. 10. Retrieved from http://www. statcan.gc.ca/pub/89-621-x/89-621-x2007010-eng.pdf

2. Milan A, Tran K. Blacks in Canada: a long history. Canadian Social Trends Statistics Canada - Catalogue No. 2004:11-008 Retrieved from https://sites.ualberta.ca/ jrkelly/blacksinCanada.pdf.

3. Statistics Canada. (2003). Canada's ethnocultural portrait: the changing mosaic. Statistics Canada Catalogue no. 96F0030XIE2001008.

4. Misri S. Perinatal mood/anxiety disorders: diagnosis and treatment. Indian J Psychiatry. 2010;52:S10.

5. Statistics Canada. (2011). National household survey. Retrieved from https://www12.statcan.gc.ca/nhs-enm/2011/as-sa/99-010-x/ 2011001/c-g/c-g02-eng.cfm

6. Dunne C. Black women's health matters. BCMJ. 2020;62(6):203-4 Retrieved from https://bcmj.org/premise/black-womens-healthmatters.

7. Woodgate RL, Busolo DS, Crockett M, Dean RA, Amaladas MR, Plourde PJ. A qualitative study on African immigrant and refugee families' experiences of accessing primary health care services in Manitoba, Canada: it's not easy! Int J Equity Health. 2017;16(1):5. https://doi.org/10.1186/s12939-016-0510-x.

8. Chui, T., \& Maheux, H. (2011). Visible minority women. Ottawa: statistics Canada. Catalogue no. 89-503-X. Retrieved from http:// www.statcan.gc.ca/pub/89-503-x/2010001/article/11527-eng.pdf

9. Kerani R, Narita M, Lipira L, Endeshaw M, Holmes KK, Golden MR. Challenges in recruiting African-born, US-based participants for HIV and tuberculosis research. J Immigr Minor Health. 2019;21(3):533-9. https://doi.org/10.1007/s10903-018-0776-8.

10. Blanchet R, Sanou D, Nana CP, Pauzé E, Batal M, Giroux I. Strategies and challenges in recruiting black immigrant mothers for a community-based study on child nutritional health in Ottawa, Canada. J Immigr Minor Health. 2017;19(2):367-72. https://doi.org/10.1007/s10903-016-0536-6.

11. Fête M, Aho J, Benoit M, Cloos P, Ridde V. Barriers and recruitment strategies for precarious status migrants in Montreal Canada. BMC Med Res Methodol. 2019;19:41. https://doi.org/10.1186/ s12874-019-0683-2.

12. Katigbak C, Foley M, Robert L, Hutchinson MK. Experiences and lessons learned in using community-based participatory research to 
recruit Asian American immigrant research participants. J Nurs Scholarsh. 2016;48(2):210-8. https://doi.org/10.1111/jnu.12194.

13. Omenka OI, Watson DP, Hendrie HC. Understanding the healthcare experiences and needs of African immigrants in the United States: a scoping review. BMC Public Health. 2020;20(1): 27. https://doi.org/10.1186/s12889-019-8127-9.

14. Mosavel M. Health promotion and cervical cancer in South Africa: why adolescent daughters can teach their mothers about early detection. Health Promot Int. 2012;27(2):157-66. https://doi.org/10. 1093/heapro/dar014.

15. Mosavel M, Ports KA. Upward communication about cancer screening: adolescent daughter to mother. J Health Commun. 2015;20(6):680-6. https://doi.org/10.1080/10810730.2015. 1012245.

16. Hussak LJ, Cimpian A. Memory accessibility shapes explanation: testing key claims of the inheritance heuristic account. Mem Cogn. 2018;46:66-88. https://doi.org/10.3758/s13421-017-0746-8.

17. Lara-Cinisomo S, Clark CT, Wood J. Increasing diagnosis and treatment of perinatal depression in Latinas and African American women: addressing stigma is not enough. Womens Health Issues. 2018;28:201-4. https://doi.org/10.1016/j.whi.2018.01.003.

18. Khanlou N, Haque N, Skinner A, Mantini A, Kurtz Landy C. Scoping review on maternal health among immigrant and refugee women in canada: prenatal, intrapartum, and postnatal care. J Pregnancy. 2017;5:1-14. https://doi.org/10.1155/2017/8783294.

19. Guerin B, Guerin P, Diiriye RO, Yates S. Somali conceptions and expectations concerning mental health: some guidelines for mental health professionals. New Zeal J Psychol. 2004;33(2):59-67.

20. Kuroczycka, A., and Vallianatos, H.. The migrant maternal: "birthing" new lives abroad. Demeter Press (2016)
21. Anaman-Torgbor JA, King J, Correa-Velez I. Barriers and facilitators of cervical cancer screening practices among African immigrant women living in Brisbane Australia. J Eur Oncol Nurs Soc. 2017;31:22-9. https://doi.org/10.1016/j.ejon.2017.09.005.

22. Cudjoe J, Turkson-Ocran RA, Ezeigwe AK, Commodore-Mensah Y, Nkimbeng M, Han HR. Recruiting African immigrant women for community-based cancer prevention studies: lessons learned from the AfroPap study. J Community Health. 2019;44(5):1019 26. https://doi.org/10.1007/s10900-019-00677-y.

23. Fryer CS, Passmore SR, Maietta RC, Petruzzelli J, Casper E, Brown NA, et al. The symbolic value and limitations of racial concordance in minority research engagement. Qual Health Res. 2016;26(6):830-41. https://doi.org/10.1177/1049732315575708.

24. Manohar N, Liamputtong P, Bhole S, Arora A. Researcher positionality in cross-cultural and sensitive research. In: Liamputtong P, editor. Handbook of Research Methods in Health Social Sciences. Singapore: Springer; 2017. https://doi.org/10. 1007/978-981-10-2779-6 35-1.

25. Sutherland MA, Collins Fantasia H. Successful research recruitment strategies in a study focused on abused rural women at risk for sexually transmitted infections. J Midwifery Womens Health. 2012;57:381-5. https://doi.org/10.1111/j.1542-2011.2011.00134. $\mathrm{x}$.

26. Suwankhong D, Liamputtong P. Cultural insiders and research fieldwork: case examples from cross-cultural research with Thai people. Int J Qual Methods. 2015;14:160940691562140. https:// doi.org/10.1177/1609406915621404.

Publisher's Note Springer Nature remains neutral with regard to jurisdictional claims in published maps and institutional affiliations. 